\title{
Deep Learning Approach Using Diffusion-Weighted Imaging to Estimate the Severity of Aphasia in Stroke Patients
}

\author{
Soo Jeong, ${ }^{\text {a,* }}$ Eun-Jae Lee, ${ }^{\text {a,* }}$ Yong-Hwan Kim, ${ }^{\mathrm{b}}$ Jin Cheol Woo, ${ }^{\mathrm{c}}$ On-Wha Ryu, ${ }^{\mathrm{a}}$ Miseon Kwon, ${ }^{\mathrm{a}}$ \\ Sun U Kwon, , Jong S. Kim, ${ }^{\text {a Dong-Wha Kang }}{ }^{\text {a }}$ \\ a'Department of Neurology, Asan Medical Center, University of Ulsan College of Medicine, Seoul, Korea \\ ${ }^{b}$ Nunaps Inc., Seoul, Korea \\ 'Asan Institute for Life Sciences, Asan Medical Center, University of Ulsan College of Medicine, Seoul, Korea
}

Background and Purpose This study aimed to investigate the applicability of deep learning (DL) model using diffusion-weighted imaging (DWI) data to predict the severity of aphasia at an early stage in acute stroke patients.

Methods We retrospectively analyzed consecutive patients with aphasia caused by acute ischemic stroke in the left middle cerebral artery territory, who visited Asan Medical Center between 2011 and 2013. To implement the DL model to predict the severity of post-stroke aphasia, we designed a deep feed-forward network and utilized the lesion occupying ratio from DWI data and established clinical variables to estimate the aphasia quotient (AO) score (range, 0 to 100) of the Korean version of the Western Aphasia Battery. To evaluate the performance of the DL model, we analyzed Cohen's weighted kappa with linear weights for the categorized $A O$ score $(0-25$, very severe; $26-$ 50, severe; $51-75$, moderate; $\geq 76$, mild) and Pearson's correlation coefficient for continuous values.

Results We identified 225 post-stroke aphasia patients, of whom 176 were included and analyzed. For the categorized AQ score, Cohen's weighted kappa coefficient was 0.59 (95\% confidence interval $[\mathrm{Cl}], 0.42$ to $0.76 ; P<0.001)$. For continuous $\mathrm{AO}$ score, the correlation coefficient between true $\mathrm{AQ}$ scores and model-estimated values was $0.72(95 \% \mathrm{Cl}, 0.55$ to $0.83 ; P<0.001)$.

Conclusions DL approaches using DWI data may be feasible and useful for estimating the severity of aphasia in the early stage of stroke.

Keywords Stroke; Aphasia; Magnetic resonance imaging; Deep learning

\author{
Correspondence: Dong-Wha Kang \\ Department of Neurology, Asan Medical \\ Center, University of Ulsan College of \\ Medicine, 88 Olympic-ro 43-gil, Songpa- \\ gu, Seoul 05505, Korea \\ Tel: +82-2-3010-3440 \\ Fax: +82-2-474-4691 \\ E-mail:dwkang@amc.seoul.kr \\ https://orcid.org/0000-0002-2999-485X \\ Received: June 8, 2021 \\ Revised: September 16, 2021 \\ Accepted: October 5, 2021 \\ *These authors contributed equally to \\ the manuscript as first author.
}

\section{Introduction}

Post-stroke aphasia is a major stroke sequela and burden which significantly and negatively affects the quality of life of patients. ${ }^{1-4}$ Identifying the severity of aphasia in the early stage of stroke is crucial in predicting the prognosis of patients. 5 However, detailed examination of post-stroke aphasia during the acute phase is often difficult because of various stroke-related conditions (e.g., altered mental status, poor cooperation, confusion, and unstable medical conditions). Stroke imaging may help evaluate the severity and prognosis of aphasia; it has been suggested to be useful in estimating roughly categorized aphasia outcomes (e.g., good vs. bad outcome) after stroke; ${ }^{6-9}$ however, detailed aphasia characteristics (e.g., continuous 
score value) have yet to be estimated from stroke imaging analysis.

Deep learning (DL) techniques, which are applications of artificial intelligence, have recently emerged and are now rigorously applied in the medical field, especially in outcome prediction studies using imaging data. ${ }^{10,11}$ Because DL techniques may use multiple features that are invisible to humans, ${ }_{1}^{12,13}$ we hypothesized that DL methods may be helpful in estimating the severity of aphasia at an acute stage after stroke using magnetic resonance imaging (MRI) data. Consequently, we developed a DL model using diffusion-weighted imaging (DWI) to estimate the aphasia quotient $(A Q)$ score of the Korean version of the Western Aphasia Battery (K-WAB) (range, 0 to 100), ${ }^{14}$ which reflects the severity of aphasia in stroke patients with acute stroke, and evaluated its performance by comparing it with the true values of each patient. In the meantime, we also developed logistic regression models that estimate the $A Q$ score to evaluate the performance of the DL method, as compared to that of the conventional machine-learning approach.

\section{Methods}

\section{Data availability}

Anonymized data are available on reasonable request from any qualified investigator.

\section{Study design and patient selection}

We retrospectively analyzed consecutive acute stroke patients with aphasia who visited the Asan Medical Center (Seoul, South Korea) within 7 days of symptom onset between January 2011 and December 2013. Aphasia was defined as the presence of a score greater than 1 in the best language category of the National Institutes of Health Stroke Scale. ${ }^{15}$ In Asan Medical Center, during the study period, we usually performed MRI, including DWI and fluid-attenuated inversion recovery sequences, in stroke patients. If patients were considered candidates for acute revascularization, brain computed tomography was the a priori imaging modality in Asan Medical Center to exclude the presence of hemorrhage; MRI was subsequently performed in the emergency room immediately after the initiation of alteplase injection. The choice to perform intra-arterial thrombolysis/thrombectomy was determined according to a comprehensive analysis considering MRI lesion characteristics, vessel status, and patients' neurological status. Therefore, DWI was performed after the initiation of alteplase before mechanical thrombectomy. After neurological stabilization at the discretion of the attending physicians, the K-WAB was routinely performed in patients with aphasia, unless patients were vitally unstable or uncooperative.

We included patients who had ischemic lesions in the left middle cerebral artery (MCA) territory and those who underwent K-WAB within 14 days of symptom onset. Patients with multiple lesions in multiple vascular territories were also included if they had aphasia and stroke lesions in the left MCA territory. Only patients who used Korean as their first language were included. We excluded patients who did not undergo DWI or who demonstrated old stroke lesions within the left MCA territory on fluid-attenuated inversion recovery or gradient echo sequences of MRI. ${ }^{16}$ Patients who had previously been diagnosed with dementia that caused communication difficulties before the index stroke were also not included in the study. In addition, left-handed patients were excluded to ensure study consistency.

\section{DWI data processing}

The patients underwent 1.5-T MRI (Magnetom Avanto, Siemens Healthineers, Erlangen, Germany). The ischemic lesion mask was extracted in the native DWI space using the FSLView toolbox in the functional MRI of the brain software library (FSL, developed by Oxford Center for Functional MRI of the Brain, Oxford, UK). Initial DWI was used for the analysis. A stroke neurologist, who was blinded to all clinical information, manually segmented the DWI high-signal intensity area to measure the ischemic lesion. In patients with lesions in multiple vascular territories, lesions outside the left MCA territory were also included in the lesion volume analysis. When we obtained image features, only lesions in the left hemisphere were targeted and analyzed. Affine transformation and nonlinear warping coefficients were estimated between the DWI using a $b$-value of zero in the native space and standard Montreal Neurological Institute 152 T2 template images via the Functional Magnetic Resonance Imaging of the Brain's linear or nonlinear image registration tool. The estimated parameters were applied to the delineated lesion masks.

To cover the whole brain area and to consider white matter regions, we used five brain ATLAS templates (Broadmann's Area [BA], Automated Anatomical Labeling [AAL], Harvard-0xford [HO], JHU white matter label [WM-Label], and JHU white matter track [WM-Track]) for analyzing the brain imaging information. The lesion occupying ratio in each individual cortex was calculated for five human brain ATLASs (BA, 41 left hemisphere regions among 82 region labels; $A A L, 54$ among 116; HO, 55 among 110; WM-Label, 19 among 44; and WM-Track, nine out of 20) in the standard Montreal Neurological Institute space to quantitatively calculate the regional damage as follows: (volume of lesion inside the individual gyrus/total volume of indi- 
vidual gyrus) $\times 100(\%) .^{17}$

\section{Data collection}

To develop the DL model, we obtained 184 features, including 178 lesion occupying ratio features derived from manually segmented infarct lesions on DWI, and six clinical features (age, sex, interval between the K-WAB test and onset, interval between DWI and onset, education, and lesion volume), which are regarded as important prognostic factors for post-stroke aphasia. ${ }^{18}$ Clinical features were obtained from electronic medical records. As an outcome parameter, the $A Q$ score, a composite score of the K-WAB, was adopted and collected. In Asan Medical Center, the K-WAB was performed by two dedicated speech therapists with experience in speech evaluation and therapy for more than 5 years. The $A Q$ score ranges from 0 to 100 , with a higher score representing better language performance.

\section{Deep learning model}

We utilized a deep feed-forward network (DFFN) for the DL model and did not use convolutional neural network approaches. We chose 178 lesion occupying ratio features associated with the left hemisphere regions among various atlases on DWI and clinical features as input parameters. DFFN was implemented using PyTorch (developed by Facebook's Al Research lab). DFFN consists of four fully connected layers: an input layer (184 nodes), 1st hidden layer (90 nodes), 2nd hidden layer (30 nodes), and an output layer. The hard hyper-tangent activation function was adopted for the 1 st and 2 nd hidden layers with minimum/maximum values of -1 to 1 . Finally, the output layer is fully connected and concatenates the outputs of the 1st and 2nd layers, resulting in a final score with a hyper-tangent activation function having a range of 0 to 1 . During training of the model, mean-squared error as a loss function and Adam optimizer with a learning rate of 0.001 with default parameters (beta $=0.9$ and 0.999 ; eps $=1 \mathrm{e}-08$; weight decay $=0$; AMSgrad=false) were adopted with $50 \%$ of dropout nodes on the 1st and 2nd hidden layers. A mini-batch of 50 samples for an epoch was used for the training, and the training was stopped when the loss from the validation data was no longer minimized compared to the best loss over 50 epochs. The maximum number of training epochs was limited to 500 .

For feature selection, we adopted a dropout technique, a regularization method to overcome redundancies in image features, and overfitting. ${ }^{19}$ A schematic diagram of the DFFN structure is shown in Supplementary Figure 1. To develop the DL model, we allocated patients admitted before January 2013 to the training set, and the remaining patients were assigned to the test set. The ratio of patients in the training and test sets was 3:1. The DL model was established with randomly selected $70 \%$ of the training set at 1,000 times (i.e., 1,000 DL models were generated by the bagging strategy). For the test set, the predicted scores from 1,000 models were averaged.

\section{Logistic regression model}

We developed a conventional machine-learning model using logistic regression with Lasso (least absolute shrinkage and selection operator) regularization. ${ }^{20}$ Lasso regression is a model that shrinks regression coefficients towards zero, thereby effectively reducing feature redundancies in the imaging features, driven by using multiple brain ATLAS templates. We used a 5-fold cross-validation to yield the optimal regularization parameter. To examine whether the logistic regression model can process a large number of image features, as well as a small number of clinical features, to improve its performance with increasing datasets, we developed multiple regression models by inputting different sets of features: (1) clinical features only, (2) imaging features only, and (3) clinical+imaging features.

\section{Statistical analysis}

Baseline characteristics between the training and test sets were compared using the chi-square test or Fisher's exact test for categorical variables and the t-test or Mann-Whitney $U$ test for continuous variables, as appropriate. To compare the voxel-wise frequency difference of lesions between the training and test groups, the Bernoulli model-based two-sample t-test was performed for each voxel. ${ }^{21}$ We calculated Cohen's weighted kappa with linear weights ${ }^{22}$ between the categorized model-estimated scores and true $\mathrm{AO}$ scores $(0-25$, very severe; $26-50$, severe; $51-75$, moderate; $\geq 76$, mild). ${ }^{23}$ The degree of agreement using Cohen's weighted kappa were as follows: poor $(<0.20)$, fair (0.21-0.40), moderate (0.41-0.60), good (0.61-0.80), and very good (0.81-1.00). In addition, we estimated the Pearson's correlation coefficients between the model-estimated and true values of the continuous AQ scores. Interpretations of the correlation coefficient $(r)$ were as follows: very weak $(0.00<|r|<0.20)$, weak $(0.20 \leq|r|<0.40)$, moderate $(0.40 \leq|r|<0.60)$, strong $(0.60 \leq|r|<0.80)$, and very strong $(0.80 \leq|r|<1.00)$. We further evaluated the correlation coefficients between model-estimated and true values of sub-domain scores of the $\mathrm{AO}$ score (spontaneous speech, comprehension, repetition, and naming). ${ }^{14}$ Patients showing notable discrepancies between model-estimated and true $\mathrm{A} 0$ scores were defined as those showing studentized residuals larger than 2 (in absolute value). ${ }^{24} \mathrm{~A}$ two-sided $P<0.05$ indicated statistical significance. All statistical analyses were performed using the 
SPSS statistical software version 25.0 (IBM Co., Armonk, NY, USA).

\section{Standard protocol approvals, registrations, and patient consents}

The study was performed in accordance with the Good Clinical Practice guidelines and the Declaration of Helsinki, and was approved by the Institutional Review Board of Asan Medical Center (IRB No. 2020-1794). Written informed consent was waived owing to the retrospective nature of the study.

\section{Results}

\section{Participants}

During the study period, a total of 225 acute stroke patients with aphasia visited Asan Medical Center and were included in the study. The K-WAB test was performed for all patients. Of these, 49 were excluded (no available DWI data [ $n=16]$, crossed aphasia with right MCA lesions $[n=7]$, old stroke lesion in the left MCA territory $[n=13]$, left-handedness $[n=10]$, and delayed K-WAB test [ $>14$ days after symptom onset, $n=3]$ ) (Figure 1 ). The included patients had a mean age of $66.5 \pm 11.8$ years, mean time interval from onset to MRI of $2.4 \pm 2.7$ days, and mean time interval from onset to $K-W A B$ of $3.8 \pm 2.7$ days. $A$

225 Consecutive acute stroke patients with aphasia (2011. 1. 1-2013. 12. 31)

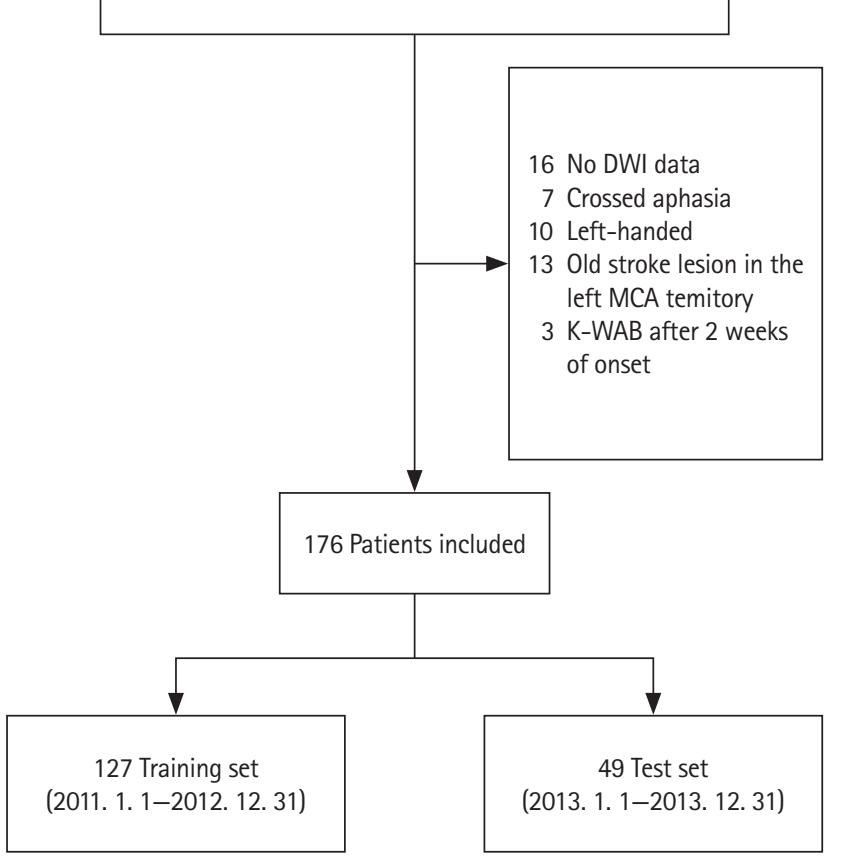

Figure 1. Flowchart showing patient selection. DWI, diffusion-weighted image; MCA, middle cerebral artery; K-WAB, Korean version of the Western Aphasia Battery. total of 36 patients received alteplase or intra-arterial thrombolysis/thrombectomy treatment. Of the 176 patients who were finally included, 127 were assigned to the training set and 49 were assigned to the test set. There were no significant differences in the baseline characteristics between the training and test sets (Table 1). The spatial patterns of lesions were analyzed by comparing the lesion proportion in every single voxel between the training and test groups using the Bernoulli model-based two-sample t-test. There were no significant differences between the groups (Figure 2).

\section{Performance of the machine-learning model}

For categorized $A Q$ score, the $D L$ model showed an accuracy of $61 \%$ in total, with Cohen's weighted kappa of 0.59 (95\% confidence interval [Cl], 0.42 to 0.76 ; $P<0.001$ ) (Table 2). In addition, the DL model showed an accuracy of $50 \%$ or more in all severity categories. The largest figure (73\%) was observed in the very severe aphasia group.

For continuous $\mathrm{A} Q \mathrm{Q}$ score, the correlation coefficient between the true $A Q$ score and model-estimated $A Q$ score was 0.72 (95\% Cl, 0.55 to $0.83 ; P<0.001)$ (Figure 3). For sub-domain scores of the AQ score, the DL model showed competent performance with strong correlation coefficient values in all categories as follows: spontaneous speech $(\mathrm{r}=0.75 ; 95 \% \mathrm{Cl}, 0.59$ to 0.85 ; $P<0.001)$; comprehension $(\mathrm{r}=0.71 ; 95 \% \mathrm{Cl}, 0.54$ to 0.83 ; $P<0.001)$; repetition $(r=0.65 ; 95 \% \mathrm{Cl}, 0.44$ to $0.78 ; P<0.001)$; and naming ( $\mathrm{r}=0.71 ; 95 \% \mathrm{Cl}, 0.54$ to $0.83 ; P<0.001)$ (Supplementary Figure 2).

We performed an additional analysis using logistic regression to evaluate the performance of the DL method, as compared to that of the conventional machine-learning approach (Supplementary Tables 1 and 2). In this process, we developed multiple regression models to determine whether a conventional model could handle large data such as image features by including different sets of input features. Consequently, logistic regression with clinical variables (only six features) showed a greater performance (correlation coefficient for $A Q$ scores, 0.70 ), as compared to the large feature situation (178 image features only, $0.54 ; 178$ image features +6 clinical features together, 0.63 ) in the test set (Supplementary Table 2).

\section{Cases with discrepancy and those with acute intervention}

Three patients demonstrated notable discrepancies between the model-estimated and true $\mathrm{AQ}$ scores (Table 3 and Figure 3 ). Cases 1 and 2 showed more severe aphasia in their K-WAB tests compared to the results predicted by the DL model, while Case 3 demonstrated opposite results with milder aphasia in 
Table 1. Baseline characteristics of patients in the training and test set

\begin{tabular}{|c|c|c|c|}
\hline Characteristic & 127 Training set & 49 Test set & $P$ \\
\hline Age (yr) & $65.9 \pm 11.8$ & $68.0 \pm 12.0$ & NS \\
\hline Female sex & $46(36.2)$ & $14(28.6)$ & NS \\
\hline Hypertension & $60(47.2)$ & $31(63.3)$ & NS \\
\hline Diabetes & 40 (31.5) & $14(28.6)$ & NS \\
\hline NIHSS on admission & $8.3 \pm 5.5$ & $8.4 \pm 5.5$ & NS \\
\hline Years of education & $10.1 \pm 5.1$ & $10.1 \pm 4.7$ & NS \\
\hline Lesion volume $\left(\mathrm{cm}^{3}\right)$ & $58.4 \pm 75.7$ & $38.9 \pm 49.9$ & NS \\
\hline MRI from onset (hr) & $56.1 \pm 66.7$ & $60.4 \pm 59.2$ & NS \\
\hline K-WAB from onset (day) & $3.9 \pm 3.0$ & $3.4 \pm 2.1$ & NS \\
\hline AQ score & $43.6 \pm 30.3$ & $37.7 \pm 31.1$ & NS \\
\hline Mild ( $\geq 76)$ & $29(22.8)$ & $13(26.5)$ & NS \\
\hline Moderate (51-75) & $28(22.0)$ & $6(12.2)$ & NS \\
\hline Severe (26-50) & $27(21.3)$ & $8(16.3)$ & NS \\
\hline Very severe (0-25) & 43 (33.9) & $22(44.9)$ & NS \\
\hline
\end{tabular}

Values are presented as mean \pm standard deviation or number (\%).

NS, non-significant; NIHSS, National Institutes of Health Stroke Scale; MRI, magnetic resonance imaging; K-WAB, Korean version of the Western Aphasia Battery; $A Q$, aphasia quotient.

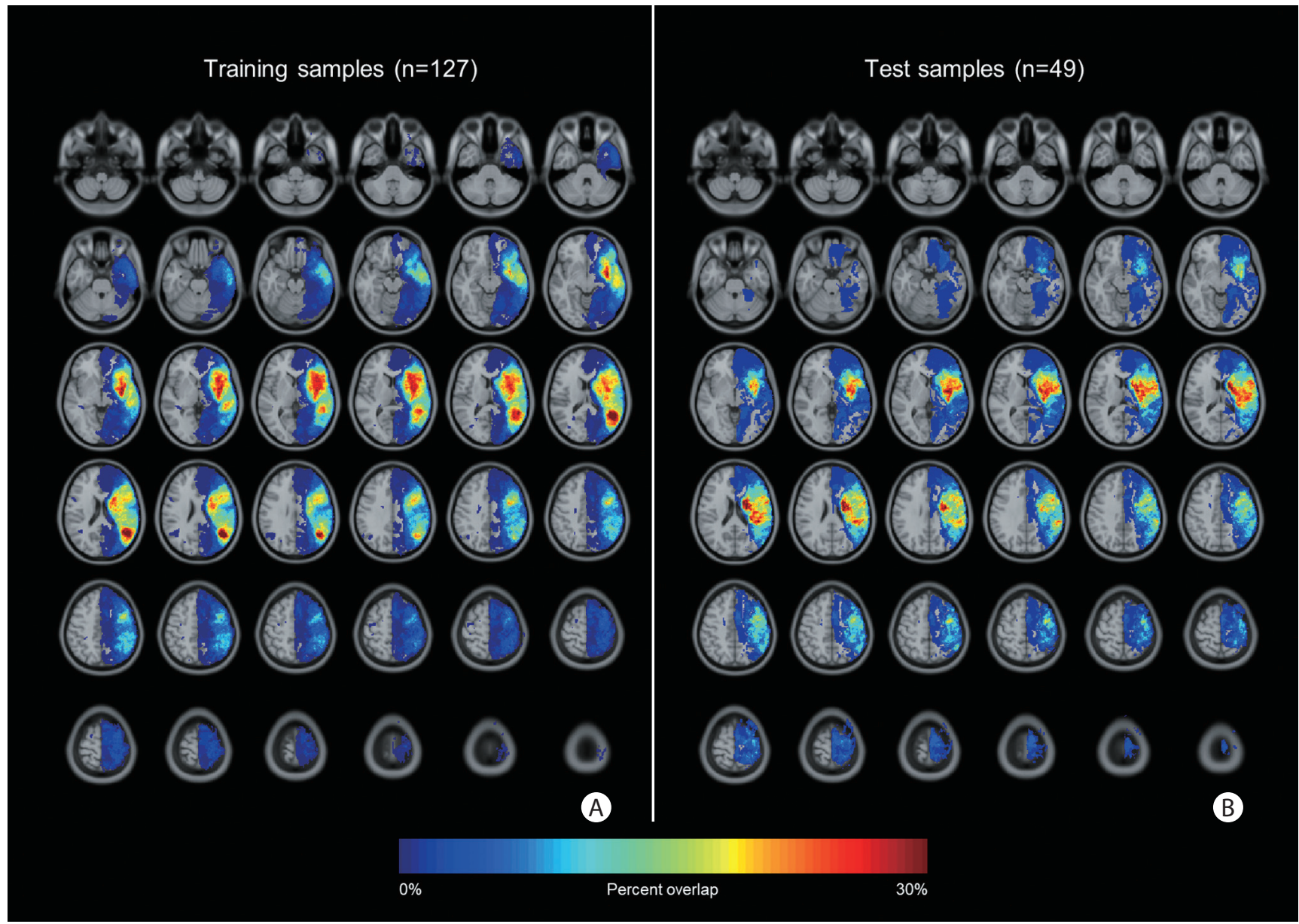

Figure 2. Lesion pattern heat maps of (A) training and (B) test groups. A heat map was used to visualize the proportion of lesions in each voxel. We compared the lesion proportion in every voxel between the training and test groups using the Bernoulli model-based two-sample t-test, but found no difference between the training and test groups ( $P>0.05)$. 
Table 2. Contingency table between the true score and model-estimated score

\begin{tabular}{|c|c|c|c|c|c|}
\hline & \multicolumn{4}{|c|}{ True score } & \multirow{2}{*}{ Total } \\
\hline & Very severe & Severe & Moderate & Mild & \\
\hline \multicolumn{6}{|c|}{ Model-estimated score } \\
\hline Very severe & 16 & 3 & 2 & 0 & 21 \\
\hline Severe & 4 & 4 & 1 & 2 & 11 \\
\hline Moderate & 1 & 1 & 3 & 4 & 9 \\
\hline Mild & 1 & 0 & 0 & 7 & 8 \\
\hline Total & 22 & 8 & 6 & 13 & 49 \\
\hline Accuracy & 16/22 (73) & 4/8 (50) & $3 / 6(50)$ & $7 / 13(54)$ & $30 / 49(61)$ \\
\hline
\end{tabular}

Values are presented as number (\%). Cohen's weighted kappa, $\mathrm{K}=0.59$ (95\% confidence interval, 0.42 to $0.76 ; P<0.001)$.

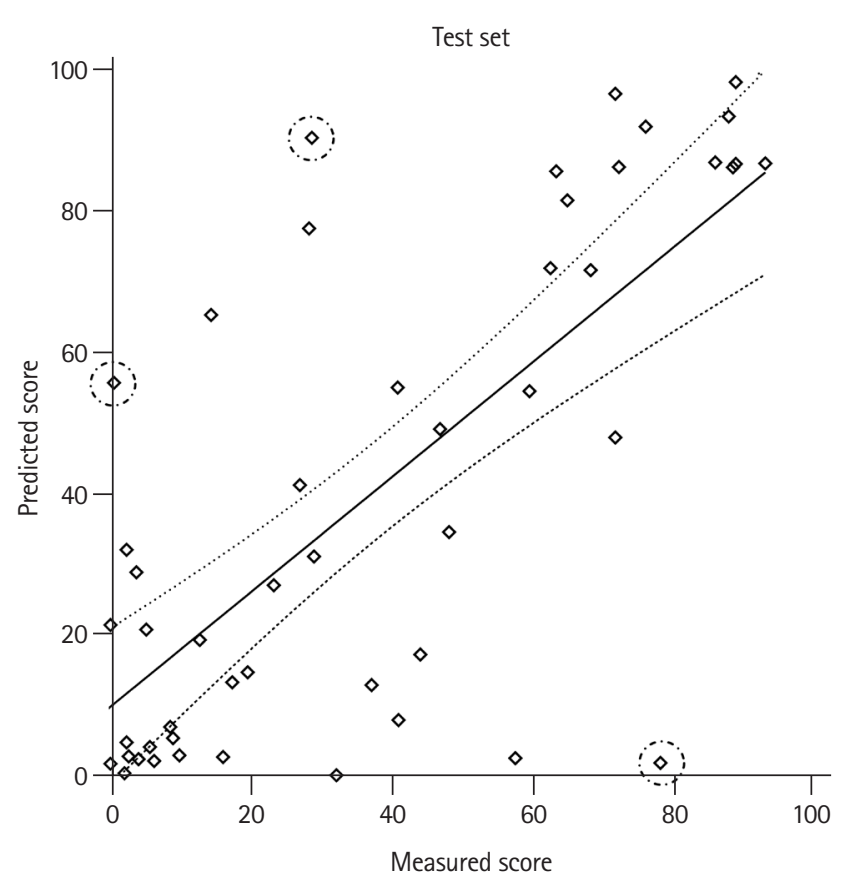

Figure 3. Correlation analysis between the true aphasia quotient (AO) score and predicted $A Q$ score in the test set. The correlation coefficient was 0.72 (95\% confidence interval, 0.55 to $0.83 ; P<0.001$ ); solid black line, regression line; dotted black line, $95 \%$ confidence limit; dotted circle, cases with notable discrepancy between the true $A Q$ score and predicted $A O$ score with studentized residual larger than 2 (in absolute value).

the K-WAB test than in the DL model. These patients had distinctive clinical features. Case 1 had a small embolic ischemic lesion on acute DWI (3.6 hours after symptom onset) with underlying left proximal internal carotid artery stenosis, which decreased perfusion in the left MCA territory. Case 2 had multiple scattered lesions that involved the bilateral frontal lobes, including the anterior cingulate cortex, on DWI (83 hours after symptom onset). Finally, Case 3 involved a patient with in-hospital stroke after endovascular coiling of a left posterior communicating artery aneurysm. This patient showed large lesions involving most of the left MCA territory on hyperacute DWI (within 1 hour after symptom onset) and subsequently underwent successful acute intra-arterial thrombectomy (Figure 4).

We subsequently evaluated Cohen's weighted kappa value and correlation coefficients only in patients who had undergone acute thrombolysis/thrombectomy. Because the number of these patients was small ( $n=24$ for the training group; $n=12$ for the test group), we combined two groups in this analysis to increase the number of patients $(n=36)$. As a result, the values of the variables $(\mathrm{K}=0.60 ; 95 \% \mathrm{Cl}, 0.41$ to $0.78 ; P<0.001)$ ( $r=0.73 ; 95 \% \mathrm{Cl}, 0.53$ to $0.85 ; P<0.001)$ were comparable to those in the total group of patients (Figure 3 and Supplementary Table 3). Notably, among the patients who underwent acute intervention, the performance of the DL model was good in patients with very severe (accuracy, 92\%) or mild (accuracy, 70\%) aphasia, while it was less remarkable in patients with moderate and severe aphasia.

\section{Discussion}

In this study, we demonstrated that DL techniques using DWI and clinical data could be used to estimate the severity of aphasia in ischemic stroke patients at an early stage. The DL model showed good performance in estimating the severity of post-stroke aphasia, as compared to the actual $A O$ score values, within 14 days after symptom onset.

Aphasia is one of the most devastating cognitive sequelae of stroke, which results in difficulties in activities of daily living in stroke patients. ${ }^{1-4}$ There are many factors that are associated with post-stroke aphasia recovery, including age, sex, handedness, lesion location and size, stroke and aphasia severity, and even non-linguistic cognitive abilities such as emotional and social characteristics. ${ }^{25}$ Among these factors, the severity of initial aphasia is one of the best predictors of aphasia outcome at a later stage. ${ }^{26-28}$ However, it is often difficult to thoroughly 
Table 3. Clinical features of cases with significant discrepancies between model-estimated score and true AQ score

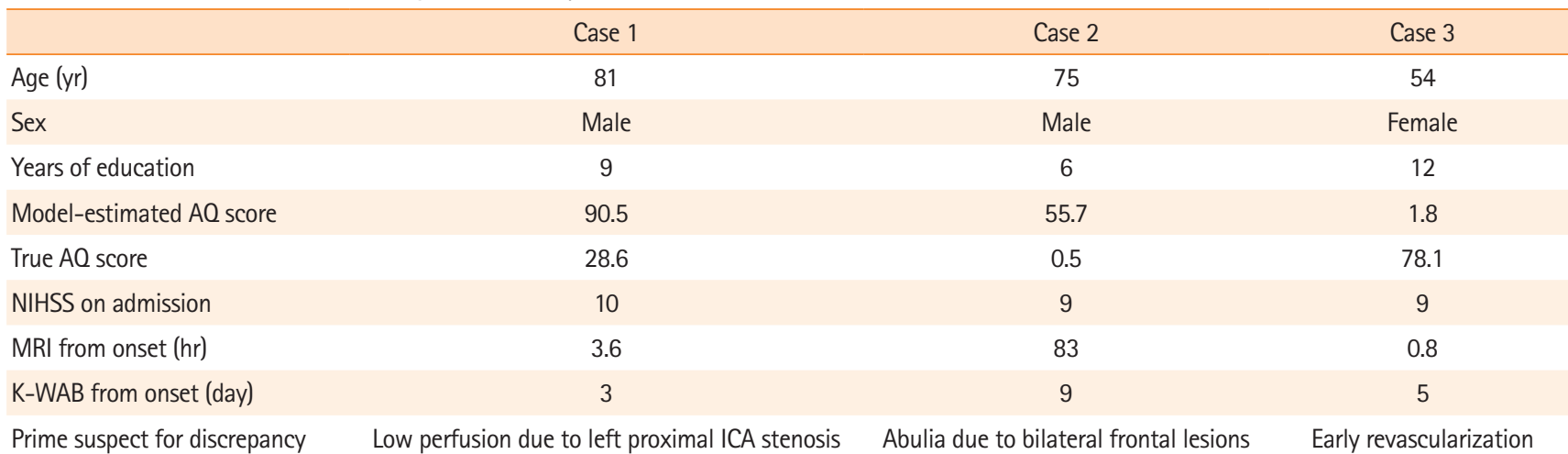

AQ, aphasia quotient; NIHSS, National Institutes of Health Stroke Scale; MRI, magnetic resonance imaging; K-WAB, Korean version of the Western Aphasia Battery; ICA, internal carotid artery.
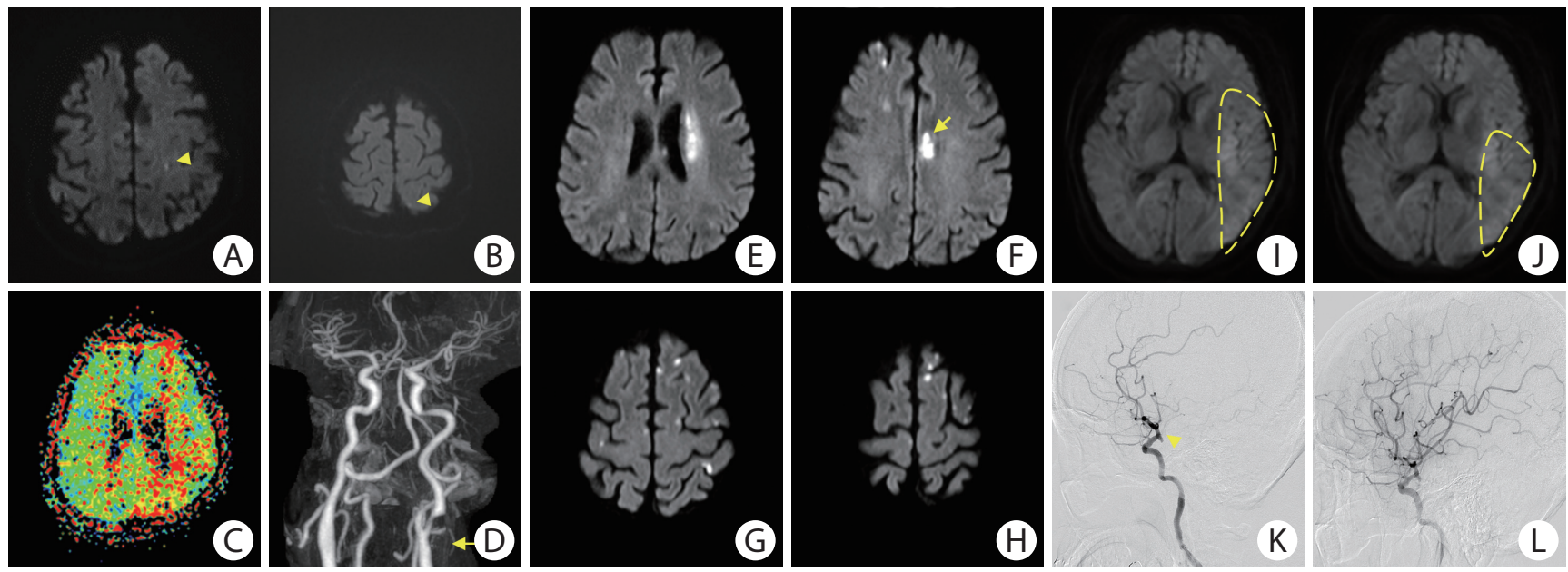

Figure 4. Imaging characteristics of cases with notable discrepancies. Panels represent (A-D) Case 1, (E-H) Case 2, and (I-L) Case 3, respectively. (A, B) Small acute lesions in the left internal carotid artery (ICA) border zone (yellow arrowheads) on diffusion-weighted imaging (DWI); (C) increased time-to-peak value in the left middle cerebral artery (MCA) territory; (D) severe stenosis in the left proximal ICA (yellow arrow); (E-H) acute infarction in the left corona radiata and bilateral anterior cerebral artery territory on DWI, especially in the left anterior cingulate cortex (yellow arrow); (I, J) large but subtle DWI high-signal intensity in the left MCA territory (yellow dashed lines); (K) occlusion of the left MCA inferior division (yellow arrow) on conventional angiography; (L) subsequent recanalization after immediate mechanical thrombectomy.

examine language function in stroke patients during the acute stage. Therefore, a DL model using acute MRI data may be useful in clinical practice because it can evaluate language function regardless of patient cooperation and/or concomitant medical conditions.

The major strength of this study is that we developed a DL model to estimate aphasia severity in detail. Although several models have been used to predict the outcome of aphasia after stroke, ${ }^{7,9,29,30}$ these models use conventional logistic regression to construct a model that uses only categorized image information (e.g., small or large lesions) or outcomes (e.g., good or bad outcomes). This process could have caused a marked loss of information. However, DL techniques can handle original data without significant modification, resulting in only a mod- est loss of information. Another strength of the DL model is its ability to handle large datasets. Although logistic regression approaches can also give rise to a model dealing with image features, the overfitting issue is always a concern in conventional models. In this study, we developed a logistic regression model, showing a comparable performance with our DL model, with only six clinical features (correlation coefficients for $\mathrm{AQ}$ scores, 0.70 vs. 0.72 ). However, logistic regression approaches failed to enhance performance when adding image features to clinical features. This unstable performance of logistic regression models raises the importance of $\mathrm{DL}$ algorithms, which can successfully process large-sized image features. ${ }^{31}$ Additionally, in this study, we used imaging (DWI lesions) data as an input without categorization, and we developed a DL model that 
could estimate not only the total $\mathrm{AQ}$ score (continuous variable), but also the sub-domain scores of language function. The resulting performance is noteworthy. The DL model could estimate the categorized $A Q$ score with moderate agreement $(\mathrm{K}=0.59)$ and continuous $\mathrm{AQ}$ score with strong correlation $(r=0.72, P<0.001)$. More detailed input data can improve model performance, while more detailed outputs can provide more information to clinicians and patients.

We noticed three patients with notable discrepancies between the model-estimated and true AO scores (Table 3 and Figure 3). Case 1 presented with a few dot-like DWI lesions in the left internal carotid artery border zone territory on acute DWI with perfusion delay in the MCA area due to proximal internal carotid artery stenosis. Decreased perfusion and subsequent lesions in the language cortex may have resulted in severe aphasia in the true $A Q$ score of the K-WAB test. However, we could not check the final lesions because follow-up MRI was not available for this patient. Case 2 exhibited scattered lesions in both anterior cerebral arterial territories involving the left anterior cingulate cortex, and the lesions and network disruption have been shown to be associated with apathy or an abulic state. ${ }^{32,33} \mathrm{~A}$ decreased responsiveness to language tasks may have resulted in a poor K-WAB test. Alternatively, we should also consider that other unknown combined conditions not captured in the medical records (e.g., fluctuating delirium) may have affected the results. Case 3 underwent DWI immediately after symptom onset and early revascularization (onset to needle time, 45 minutes). Although we did not perform follow-up MRI, we speculated that acute lesions on initial DWI may have been reversed with neurological recovery. Reversible acute DWI lesions in patients thrombolyzed within 4.5 hours have been reported, and they are associated with early neurological improvement. ${ }^{34}$ Taken together, these cases suggest that our DL model results should be interpreted individually according to each patient's clinical situation.

The $D L$ technique also estimated the $A 0$ score of patients who received acute thrombolysis/thrombectomy in our cohort. This is remarkable in that MRI findings at a time point early enough to perform acute interventions may also be useful in estimating the degree of aphasia at an early stage. However, the performance of the DL model in an acute intervention setting is likely to be reliable in patients with mild or very severe aphasia, but not in those with a medium degree of aphasia. Because intervention procedures may result in early neurological improvement or alteration, the model-predicted aphasia outcomes should be interpreted with caution in patients undergoing acute interventions (as in Case 3 in Table 3). Notwithstanding these aspects, the good performance of the DL model to estimate aphasia severity in many patients undergoing acute intervention may further raise the applicability of our DL technique in real-world practice.

In the present study, the DL model used a skip connection instead of a plain network (i.e., concatenated outputs of the 1st and 2nd hidden layers for input in the output layer; this is called the residual neural network). Previous studies have shown that the residual neural network architecture is useful to avoid the problem of vanishing gradients in multi-layer neural networks in case of an image recognition field. ${ }^{35,36}$ In our DL model, it was also useful to reduce the time in the convergence speeds for training. Our DL model involved 90 and 30 nodes in the 1st and 2nd hidden layers, respectively, and showed the best performance in the measurement of correlation coefficients between measured and predicted $\mathrm{AO}$ scores in our additional evaluations among the tested combinations of 10,30 , 50, 70, and 90 nodes for the 1st and 2nd hidden layers. The optimal number of nodes for the hidden layers may be limited in this study; however, our approach for DL modeling is promising for predicting $A Q$ scores.

Our study has some limitations. First, it was a retrospective observational study with a single-center cohort, and therefore has a certain level of inherent bias. Furthermore, all patients used only Korean as their first language, which may have decreased the generalizability of the study results. In addition, we did not correlate early aphasia results with long-term aphasia. Although the severity of early aphasia is an important predictor of chronic aphasia, ${ }^{5,26-28}$ most previous studies dealing with post-stroke aphasia have been dedicated to predicting the prognosis at 1 year after stroke, which is regarded as a "plateau" status. Therefore, long-term and prospective studies are warranted to broaden our results. ${ }^{7,9,29,30}$ Third, we arbitrarily allocated patients into training and test sets according to the admission time. Although the baseline characteristics were not statistically different between the two groups, a multicenter cohort study with external validation could improve the generalizability of our model. Fourth, because we used multiple brain ATLAS templates with overlaps, image features may have been calculated and derived from overlapping templates. Thus, redundancies in image-feature information may be possible. However, using multiple templates is unavoidable to cover the entire brain area related to language function. Moreover, it should also be noted that we adopted regularization methods, such as Lasso, for logistic regression and dropout approach for DFFN, to minimize these biases in feature selection. Finally, we used only DWI data for the analysis and did not consider various MRI modalities. Multiple sequences of MRI may be helpful in estimating neurological phenotypes. For example, fluid-at- 
tenuated inversion recovery and susceptibility-weighted imaging sequences show underlying old ischemic or hemorrhagic lesions; perfusion MRI demonstrates the penumbra that may predict the final infarction territory; and diffusion tensor tractography could predict the integrity of fasciculi of language domains. However, including only a simple MRI sequence may have enhanced the clinical utility of our DL model. Nevertheless, future studies to evaluate the usefulness of multiple MRI sequences in estimating the severity of aphasia are warranted.

\section{Conclusions}

Our study suggests that the DL model using DWI data may be feasible and useful in estimating the severity of aphasia in patients with acute stroke at an early stage. These findings warrant further research to evaluate the applicability of DL model in different study populations.

\section{Supplementary materials}

Supplementary materials related to this article can be found online at https://doi.org/10.5853/jos.2021.02061.

\section{Disclosure}

Yong-Hwan Kim was employed by company Nunaps Inc.

The remaining authors declare that the research was conducted in the absence of any commercial or financial relationships that could be construed as a potential conflict of interest.

\section{Acknowledgments}

This research was supported by grants from the Korea Health Technology R\&D Project through the Korea Health Industry Development Institute (KHIDI), funded by the Ministry of Health \& Welfare (HI18C2383) and the Ministry of Science and ICT (NRF-2018M3A9E8066249), Republic of Korea.

\section{References}

1. Benjamin EJ, Muntner P, Alonso A, Bittencourt MS, Callaway CW, Carson AP, et al. Heart disease and stroke statistics-2019 update: a report from the American Heart Association. Circulation 2019;139:e56-e528.

2. Ellis $C$, Dismuke $C$, Edwards KK. Longitudinal trends in aphasia in the United States. NeuroRehabilitation 2010;27:327-333.

3. Ellis C, Simpson AN, Bonilha $H$, Mauldin PD, Simpson KN. The one-year attributable cost of poststroke aphasia. Stroke
2012;43:1429-1431.

4. Charidimou A, Kasselimis D, Varkanitsa M, Selai C, Potagas C, Evdokimidis I. Why is it difficult to predict language impairment and outcome in patients with aphasia after stroke? J Clin Neurol 2014;10:75-83.

5. Pedersen PM, Jørgensen HS, Nakayama H, Raaschou HO, Olsen TS. Aphasia in acute stroke: incidence, determinants, and recovery. Ann Neurol 1995;38:659-666.

6. Nouwens F, de Jong-Hagelstein M, De Lau LM, Dippel DW, Koudstaal PJ, van de Sandt-Koenderman WM, et al. Severity of aphasia and recovery after treatment in patients with stroke. Aphasiology 2014;28:1168-1177.

7. El Hachioui $H$, Lingsma HF, van de Sandt-Koenderman MW, Dippel DW, Koudstaal PJ, Visch-Brink EG. Long-term prognosis of aphasia after stroke. J Neurol Neurosurg Psychiatry 2013;84:310-315.

8. Nouwens $F$, Visch-Brink EG, El Hachioui $H$, Lingsma HF, van de Sandt-Koenderman MW, Dippel DWJ, et al. Validation of a prediction model for long-term outcome of aphasia after stroke. BMC Neurol 2018;18:170.

9. Payabvash S, Kamalian S, Fung S, Wang Y, Passanese J, Kamalian $\mathrm{S}$, et al. Predicting language improvement in acute stroke patients presenting with aphasia: a multivariate logistic model using location-weighted atlas-based analysis of admission CT perfusion scans. AJNR Am J Neuroradiol 2010;31:16611668.

10. Lee EJ, Kim YH, Kim N, Kang DW. Deep into the brain: artificial intelligence in stroke imaging. J Stroke 2017;19:277-285.

11. Lee H, Lee EJ, Ham S, Lee HB, Lee JS, Kwon SU, et al. Machine learning approach to identify stroke within 4.5 hours. Stroke 2020;51:860-866.

12. Kassner A, Thornhill RE. Texture analysis: a review of neurologic MR imaging applications. AJNR Am J Neuroradiol 2010;31:809-816.

13. Wernick MN, Yang Y, Brankov JG, Yourganov G, Strother SC. Machine learning in medical imaging. IEEE Signal Process Mag 2010;27:25-38.

14. Kim $\mathrm{H}, \mathrm{Na} \mathrm{DL}$. Normative data on the Korean version of the Western Aphasia Battery. J Clin Exp Neuropsychol 2004;26: 1011-1020

15. Brott $T$, Adams HP Jr, Olinger CP, Marler JR, Barsan WG, Biller $J$, et al. Measurements of acute cerebral infarction: a clinical examination scale. Stroke 1989;20:864-870.

16. Forsting $M$, Janen $0 . M R$ Neuroimaging: Brain, Spine, Peripheral Nerves. New York, NY: Thieme, 2017.

17. Kim BJ, Kim YH, Kim N, Kwon SU, Kim SJ, Kim JS, et al. Lesion location-based prediction of visual field improvement after cerebral infarction. PLoS One 2015;10:e0143882. 
18. Plowman E, Hentz B, Ellis C Jr. Post-stroke aphasia prognosis: a review of patient-related and stroke-related factors. J Eval Clin Pract 2012;18:689-694.

19. Srivastava N, Hinton G, Krizhevsky A, Sutskever I, Salakhutdinov R. Dropout: a simple way to prevent neural networks from overfitting. J Mach Learn Res 2014;15:1929-1958.

20. Raita Y, Goto T, Faridi MK, Brown DF, Camargo CA Jr, Hasegawa K. Emergency department triage prediction of clinical outcomes using machine learning models. Crit Care 2019;23 :64.

21. Chung MK. Statistical and Computational Methods in Brain Image Analysis. Boca Raton, FL: CRC Press, 2013.

22. Martinez-Martin P, Radicati FG, Rodriguez Blazquez C, Wetmore J, Kovacs N, Ray Chaudhuri K, et al. Extensive validation study of the Parkinson's disease composite scale. Eur J Neurol 2019;26:1281-1288.

23. Kertesz A. Western Aphasia Battery-Revised (WAB-R): Examiner's Manual. San Antonio, TX: PsychCorp, 2006.

24. Andersen R. Modern Methods for Robust Regression. Los Angeles, CA: Sage Publications, 2007.

25. Watila MM, Balarabe SA. Factors predicting post-stroke aphasia recovery. J Neurol Sci 2015;352:12-18.

26. Laska AC, Hellblom A, Murray V, Kahan T, Von Arbin M. Aphasia in acute stroke and relation to outcome. J Intern Med 2001; 249:413-422.

27. Lazar RM, Minzer B, Antoniello D, Festa JR, Krakauer JW, Marshall RS. Improvement in aphasia scores after stroke is well predicted by initial severity. Stroke 2010;41:1485-1488.

28. Glize B, Villain M, Richert L, Vellay M, de Gabory I, Mazaux $J M$, et al. Language features in the acute phase of poststroke severe aphasia could predict the outcome. Eur J Phys Rehabil
Med 2017;53:249-255.

29. Blom-Smink MR, van de Sandt-Koenderman MW, Lingsma HF, Heijenbrok-Kal MH, Ribbers GM. Predicting everyday verbal communicative ability after inpatient stroke rehabilitation in patients with moderate and severe aphasia at admission: validation of a prognostic model. Eur J Phys Rehabil Med 2019;55: 532-534.

30. Godecke E, Rai T, Ciccone N, Armstrong E, Granger A, Hankey GJ. Amount of therapy matters in very early aphasia rehabilitation after stroke: a clinical prognostic model. Semin Speech Lang 2013;34:129-141.

31. Liu X, Gao K, Liu B, Pan C, Liang K, Yan L, et al. Advances in deep learning-based medical image analysis. Health Data Sci $2021 ; 2021: 8786793$.

32. Das JM, Saadabadi A. Abulia. In: StatPearls. Treasure Island, FL: StatPearls Publishing, 2019. https://www.ncbi.nlm.nih. gov/books/NBK537093. Assessed December 1, 2021.

33. Siegel JS, Snyder AZ, Metcalf NV, Fucetola RP, Hacker CD, Shimony JS, et al. The circuitry of abulia: insights from functional connectivity MRI. Neuroimage Clin 2014;6:320-326.

34. Labeyrie MA, Turc G, Hess A, Hervo P, Mas JL, Meder JF, et al. Diffusion lesion reversal after thrombolysis: a MR correlate of early neurological improvement. Stroke 2012;43:2986-2991.

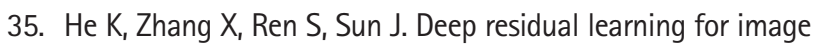
recognition. Proceedings of the 2016 IEEE Conference on Computer Vision and Pattern Recognition (CVPR); 2016 Jun 27-30; Las Vegas, NV. IEEE; 2016;770-778. https://www.computer.org/csdl/proceedings-article/cvpr/2016/8851a770/120mNxvwoXv. Assessed December 1, 2021.

36. Srivastava RK, Greff K, Schmidhuber J. Highway networks. arXiv 2015 Nov 3. https://arxiv.org/abs/1505.00387. 
Supplementary Table 1. Performance of logistic regression and deep learning methods depending on input features in the training set

\begin{tabular}{|c|c|c|c|c|c|}
\hline Performance in training set* $(n=127)$ & AQ score & Spontaneous speech & Comprehension & Repetition & Naming \\
\hline \multicolumn{6}{|l|}{ Logistic regression with LASSO } \\
\hline Clinical feature only (6 features) & 0.74 & 0.70 & 0.72 & 0.71 & 0.74 \\
\hline Imaging feature only (178 features) & 0.79 & 0.82 & 0.75 & 0.79 & 0.76 \\
\hline Clinical+imaging features & 0.84 & 0.75 & 0.82 & 0.84 & 0.80 \\
\hline \multicolumn{6}{|l|}{ Deep feed forward network } \\
\hline Clinical+imaging features & 0.86 & 0.88 & 0.84 & 0.88 & 0.85 \\
\hline
\end{tabular}

$\mathrm{AQ}$, aphasia quotient; LASSO, least absolute shrinkage and selection operator.

*Performance was calculated using the correlation coefficients between the actual and predicted Korean version of the Western Aphasia Battery (K-WAB) scores.

Supplementary Table 2. Performance of logistic regression and deep learning methods depending on the input features in the test set

\begin{tabular}{|c|c|c|c|c|c|}
\hline 49 Performance in test set ${ }^{*}(n=49)$ & AQ score & Spontaneous speech & Comprehension & Repetition & Naming \\
\hline \multicolumn{6}{|l|}{ Logistic regression with LASSO } \\
\hline Clinical feature only (6 features) & 0.70 & 0.61 & 0.70 & 0.62 & 0.66 \\
\hline Imaging feature only (178 features) & 0.54 & 0.64 & 0.54 & 0.53 & 0.50 \\
\hline Clinical+imaging features & 0.63 & 0.67 & 0.65 & 0.59 & 0.59 \\
\hline \multicolumn{6}{|l|}{ Deep feed forward network } \\
\hline Clinical+imaging features & 0.72 & 0.75 & 0.71 & 0.65 & 0.71 \\
\hline
\end{tabular}

$\mathrm{AO}$, aphasia quotient; LASSO, least absolute shrinkage and selection operator.

*Performance was calculated in the correlation coefficients between actual and predicted Korean version of the Western Aphasia Battery (K-WAB) scores.

Supplementary Table 3. Contingency table between the true and model-predicted scores in patients who underwent acute intervention

\begin{tabular}{|c|c|c|c|c|c|}
\hline & \multicolumn{4}{|c|}{ True score } & \multirow{2}{*}{ Total } \\
\hline & Very severe & Severe & Moderate & Mild & \\
\hline \multicolumn{6}{|c|}{ Model-predicted score } \\
\hline Very severe & 11 & 4 & 1 & 1 & 17 \\
\hline Severe & 1 & 1 & 3 & 0 & 5 \\
\hline Moderate & 0 & 0 & 2 & 2 & 4 \\
\hline Mild & 0 & 2 & 1 & 7 & 10 \\
\hline Total & 12 & 7 & 7 & 10 & 36 \\
\hline Accuracy & $11 / 12$ (92) & $1 / 7(14)$ & 2/7 (29) & $7 / 10$ (70) & $21 / 36(58)$ \\
\hline
\end{tabular}

Values are presented as number (\%). Cohen's weighted kappa, $\kappa=0.60$ (95\% confidence interval, 0.41 to $0.78 ; P<0.001)$. 


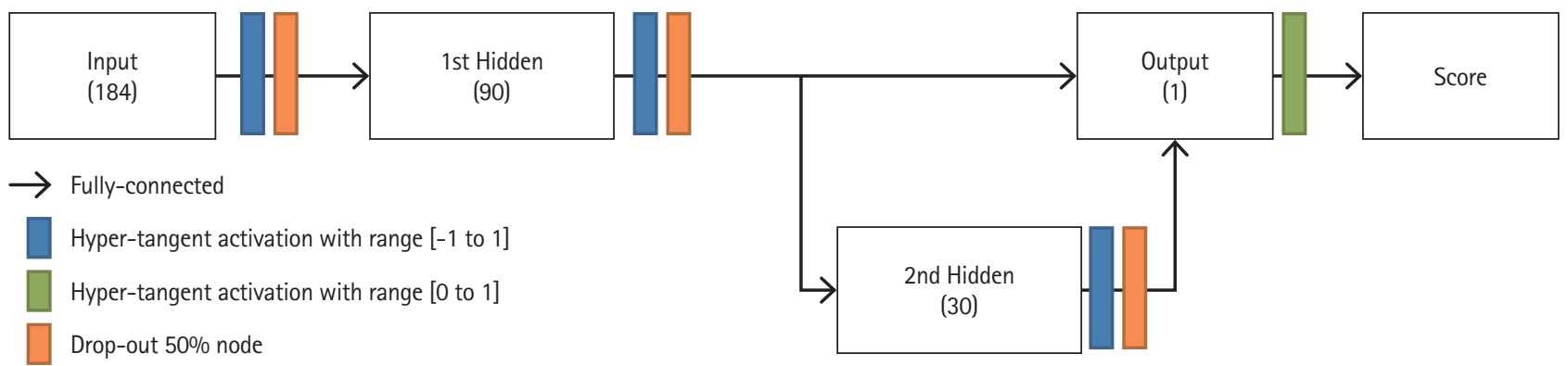

Supplementary Figure 1. Structure of the deep learning (DL) model. The number of nodes in each layer was noted in the box. For the input layer, 178 lesion occupying ratio features (left hemisphere associated regions from various atlases) and six clinical features (age, sex, Korean version of the Western Aphasia Battery [K-WAB] evaluation days from magnetic resonance imaging [MRI], MRI hours from onset, education years, and lesion volume) were used. Using the DL model, the final score was predicted to range from 0 to 1 . The true score of the K-WAB was fed into the model by normalizing scores in the range of 0 to 1.

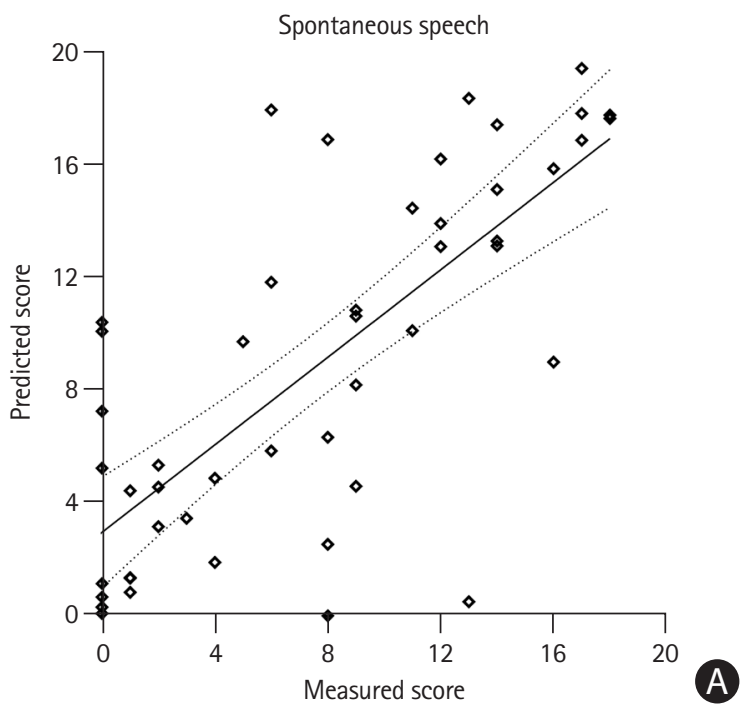

Repetition

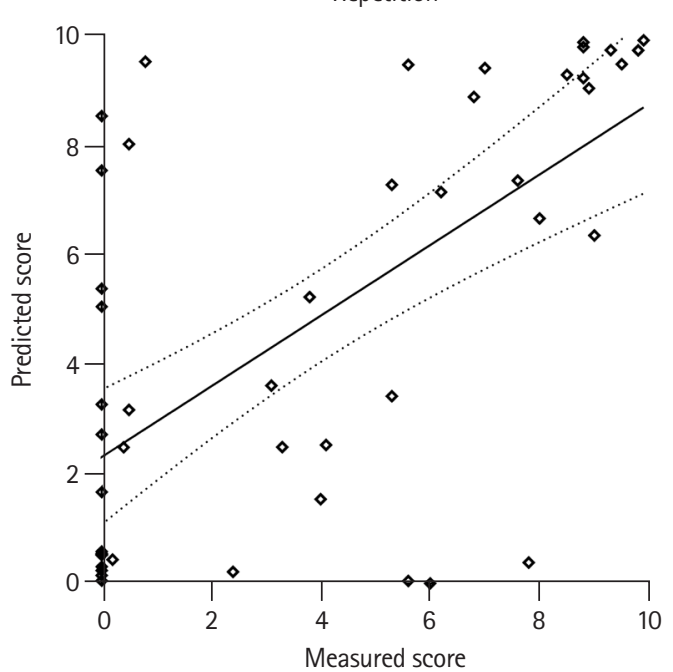

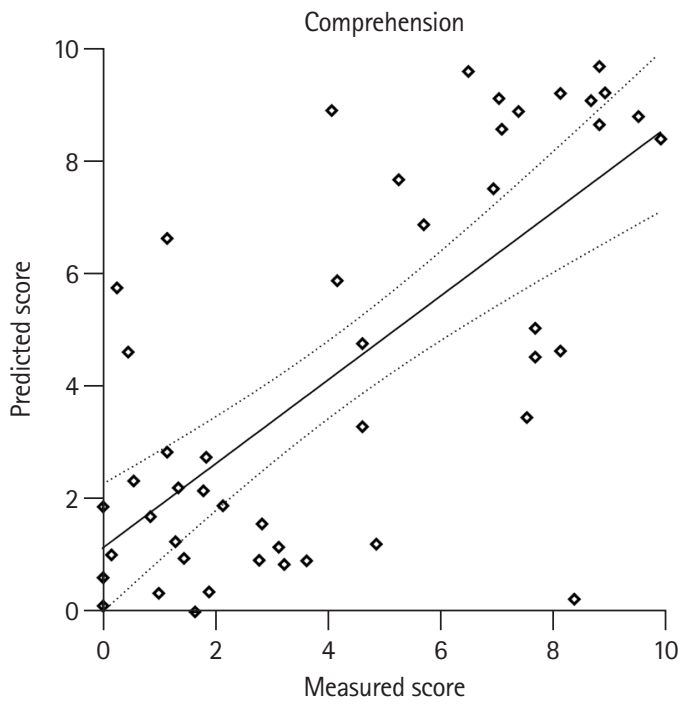

B

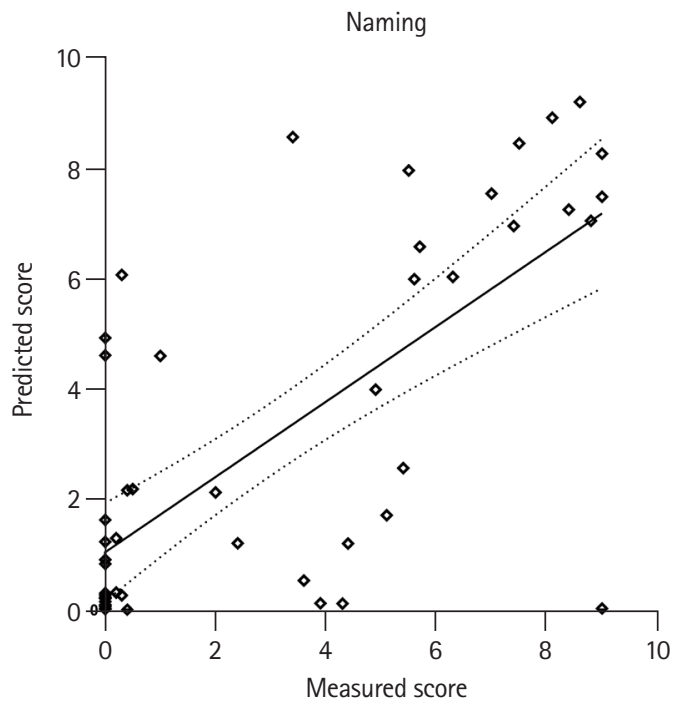

Supplementary Figure 2. Correlation analysis of sub-scores of aphasia quotient in the test set. (A) The correlation coefficient of spontaneous speech was 0.75 (95\% confidence interval $[\mathrm{Cl}], 0.59$ to $0.85 ; P<0.001)$. (B) The correlation coefficient of repetition was $0.65(95 \% \mathrm{Cl}, 0.44$ to $0.78 ; P<0.001)$. (C) The correlation coefficient of comprehension was 0.71 ( $95 \% \mathrm{Cl}, 0.54$ to 0.83 ; $P<0.001$ ). (D) The correlation coefficient of naming was 0.71 (95\% $\mathrm{Cl}, 0.54$ to 0.83 ; $P<0.001$ ) (solid black line, regression line; dotted black line, $95 \%$ confidence limit). 


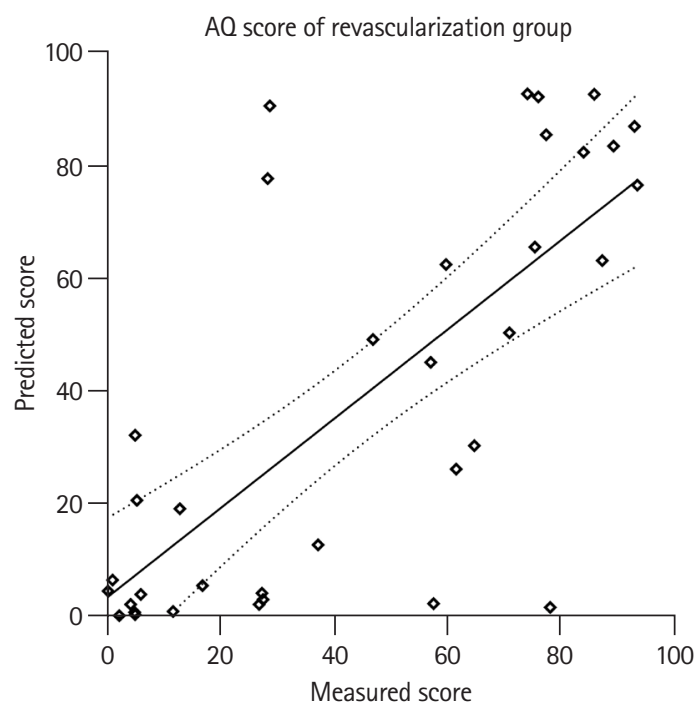

Supplementary Figure 3. Correlation analysis between true aphasia quotient (A0) score and model-predicted A0 score of stroke patients undergone acute intervention. The correlation coefficient was 0.73 (95\% confidence interval, 0.53 to $0.85 ; P<0.001$ ). 